\title{
Produção e caracterização de compósitos à base de gesso reforçado com partículas de resíduo da agroindústria do cacau
}

\section{Production and characterization of gypsum-based composites reinforced with cocoa agroindustry waste particles}

\author{
Maria Cecíllia Ramos de Araújo Veloso ${ }^{1}$, Luciana Silva Villela ${ }^{1}$, \\ Laércio Mesquita Júnior ${ }^{1}$, Mara Lúcia Agostini Valle ${ }^{2}$, Lourival Marin Mendes ${ }^{3}$, \\ José Benedito Guimarães Júnior ${ }^{4}$
}

\footnotetext{
${ }^{1}$ Departamento de Ciências Florestais - Universidade Federal de Lavras, C.P. 3037, CEP: 37200-000, Lavras, MG, Brasil

${ }^{2}$ Departamento de Engenharia Florestal - Universidade Federal do Sul da Bahia, C.P., CEP 45602-031, Itabuna, BA, Brasil

${ }^{3}$ Departamento de Engenharia Florestal - Universidade Federal de Lavras, C.P. 3037, CEP: 37200-000, Lavras, MG, Brasil

${ }^{4}$ Departamento de Engenharia - Universidade Federal de Lavras, C.P. 3037, CEP: 37200-000, Lavras, MG, Brasil e-mail: mariacraveloso@outlook.com, lucianavillela_3@hotmail.com, laerciomjr@gmail.com, maraagostini@ufsb.edu.br, lourival@dcf.ufla.br, jose.guimaraes@deg.ufla.br
}

\section{RESUMO}

Este trabalho teve como objetivo produzir e caracterizar compósitos à base de gesso reforçados com partículas de resíduo de cacau como forma de agregar valor a esse subproduto agrícola e criar um material inovador. Para a produção dos compósitos foram utilizados gesso fino para fundição e partículas de resíduo de cacau. As proporções de reforço inseridas foram de $0,0 \% ; 2,5 \% ; 5,0 \% ; 7,5 \%$ e 10,0\% em substituição a massa de gesso. Para a produção dos compósitos foram utilizadas fôrmas de madeira com dimensões de 40 x 40 x 160 $\mathrm{mm}$. Foi realizada análise da composição química do resíduo de cacau para determinação dos teores de extrativos totais, cinzas, lignina e holocelulose. Os compósitos foram avaliados nos ensaios físicos, mecânicos, térmico e acústico. Constatou-se que, houve melhorias nas propriedades físicas com o acréscimo de reforço tornando o material mais leve, visto a redução da densidade $\left(1190,69\right.$ a $\left.1138,92 \mathrm{~kg} / \mathrm{m}^{3}\right)$, e proporcionando a diminuição da higroscopicidade, com teores de umidade decrescendo de 7,74 a 4,01\% e de absorção de água decrescendo de 18,37 para 5,28\%. Da mesma forma, a resistência térmica aumentou gradativamente ao longo dos tratamentos com valores de 1,90 a $2,41 \mathrm{~K} . \mathrm{m} / \mathrm{W}$ e o isolamento acústico foi maior nos compósitos em comparação com o gesso puro, embora não tenha havido linearidade entre os tratamentos. Já para as propriedades mecânicas, houve diminuição de resistências tanto à flexão (3,60 a 0,84 MPa) quanto à compressão (4,43 a 1,73 MPa). Foi concluído que o resíduo de cacau apresenta potencialidade para ser utilizado no compósito tendo em vista que a inclusão de até $8,15 \%$ do mesmo atendeu a normatização em todos os ensaios realizados. Isso fomenta a ideia de que os resíduos lignocelulósicos são alternativas promissoras para criação de materiais de alto valor agregado.

Palavras-chave: Resíduo lignocelulósico, absorção de água, resistência térmica, materiais compósitos.

\section{ABSTRACT}

This work aimed to produce and characterize gypsum-based composites reinforced with cocoa waste parti- 
cles as a way to add value to this agricultural by-product and create an innovative material. For the production of composites, thin plaster for casting and particles of cocoa waste were used. The reinforcement proportions inserted were $0.0 \% ; 2.5 \% ; 5.0 \% ; 7.5 \%$ and $10.0 \%$ replacing plaster mass. For the production of composites, wooden molds with dimensions of $40 \times 40 \times 160 \mathrm{~mm}$ were used. Analysis of the chemical composition of the cocoa waste was performed to determine the levels of total extracts, ash, lignin and holocellulose. The composites were evaluated in physical, mechanical, thermal and acoustic tests. It was found that there were improvements in physical properties with the addition of reinforcement making the material lighter, given the reduction in density $\left(1190.69\right.$ to $\left.1138.92 \mathrm{~kg} / \mathrm{m}^{3}\right)$, and providing a decrease in hygroscopicity, with moisture levels decreasing from 7.74 to $4.01 \%$ and water absorption decreasing from 18.37 to $5.28 \%$. Likewise, the thermal resistance gradually increased over the treatments with values from 1.90 to $2.41 \mathrm{~K} . \mathrm{m} / \mathrm{W}$ and the acoustic insulation was higher in composites compared to pure plaster, although there was no linearity between treatments. As for the mechanical properties, there was a decrease in resistance both to flexion (3.60 to $0.84 \mathrm{MPa}$ ) and to compression (4.43 to $1.73 \mathrm{MPa}$ ). It was concluded that the cocoa waste has the potential to be used in the composite considering that the inclusion of up to $8.15 \%$ of the same met the standard in all tests performed. This promotes the idea that lignocellulosic waste is a promising alternative for creating materials with high added value.

Keywords: Lignocellulosic waste, water absorption, thermal resistance, composite materials.

\section{INTRODUÇÃO}

O gesso está entre os materiais de construção mais antigos que se tem conhecimento. No Brasil, sua utilização é ampla abrangendo forros, revestimentos, blocos, placas acartonadas e ornamentos pré-moldados, como molduras, faixas, placas decorativas e florões, dentre outros. De acordo com PINTO et al. [1], o consumo desse material tem apresentado crescimento principalmente devido à facilidade e a rapidez da instalação das chapas, facilidade de moldagem e excelente acabamento e decoração.

Porém, atualmente a busca pelo desenvolvimento de materiais inovadores e sustentáveis é cada vez mais crescente e isso inclui os materiais utilizados na construção civil. Uma das alternativas que têm sido estudadas para esse propósito é a incorporação de subprodutos industriais, buscando-se obter materiais menos agressivos ao ambiente, mais econômicos, duráveis e resistentes. Ainda, estudos sobre a reutilização de materiais são de grande interesse do ponto de vista ambiental e econômico, já que com a destinação correta do resíduo o impacto ambiental é reduzido e economiza-se com tratamento ou eliminação dos mesmos. Ressalta-se que essas ações contribuem para o cumprimento dos objetivos da Agenda 2030 [2] especialmente o $\mathrm{N}^{\circ} 9$, que trata da industrialização inclusiva e sustentável, e o $\mathrm{N}^{\mathrm{o}} 12$, que fornece padrões de produção e consumo sustentáveis.

Nessa temática, o compósito é uma alternativa tanto para criação de novos materiais quanto para o aproveitamento de resíduos já que se trata da mistura de dois ou mais constituintes para produção de um único produto. Muitos materiais compósitos são compostos por apenas duas fases; uma é chamada de matriz, que é contínua e envolve a outra fase, chamada frequentemente de fase dispersa [3]. A agroindústria brasileira apresenta inúmeros resíduos lignocelulósicos com potencialidades de utilização nesses materiais, seja em forma de fibras ou partículas, por se tratar de matérias primas renováveis, abundantes e com boas propriedades mecânicas.

Dessa forma, compósitos com matriz de gesso e reforço lignocelulósico já vem sendo estudados apresentando resultados interessantes como o aumento da leveza do material e melhoria de propriedades do próprio gesso como a resistência mecânica, a absorção de água e o comportamento térmico e acústico [4]. Dentre estes trabalhos, pode-se citar a produção de compósitos com inserção de fibras de palmeira [5], fibras de abaca [6] e fibras de banana e de sisal [7], por exemplo.

A indústria do cacau no Brasil ultrapassa 700.000 ha de área colhida [8]. Com essa produção em larga escala é gerado também um grande volume de resíduos. O tegumento da amêndoa do cacau, mais especificamente a testa, é um subproduto gerado nas indústrias de chocolate correspondendo à parte que envolve a amêndoa e que deve ser retirada para obtenção do líquor. De acordo com SILVA et al. [9], uma tonelada de amêndoas com $7 \%$ de umidade pode gerar de $80 \mathrm{~kg}$ a $120 \mathrm{~kg}$ de tegumento após o processamento. Devido a este elevado volume, estes são majoritariamente utilizados para queima nas próprias indústrias, vendidos como cobertura agrícola ou simplesmente descartados $[9,10]$, como normalmente é feito para os resíduos agrícolas em geral.

Alguns estudos já mostram o potencial do tegumento da amêndoa de cacau, com pré-tratamento por ex- 
tração ou bioconversão de seus componentes químicos, visando seu melhor aproveitamento. Nesse sentido, pode-se citar sua utilização em produtos para alimentação [11], como potencial antimicrobiano [12], como descontaminante ambiental [13], dentre outros. Porém, não é encontrado na literatura muitos estudos relacionados ao aproveitamento desse resíduo de forma in natura, principalmente em compósitos. Visto isso, o uso do tegumento da amêndoa do cacau para produção desses materiais pode ser considerado a fim de ampliar suas possibilidades de utilização.

Dessa forma, o objetivo deste trabalho foi produzir e caracterizar compósitos à base de gesso reforçados com partículas de resíduo de cacau como forma de agregar valor a esse subproduto agrícola e criar um material inovador.

\section{MATERIAIS E MÉTODOS}

\subsection{Obtenção e preparação do material}

O resíduo do cacau foi obtido através de indústria cacaueira do sul da Bahia e enviado à Unidade Experimental em Painéis de Madeira da Universidade Federal de Lavras, onde foi moído e peneirado em peneiras sobrepostas de 12 mesh $(1,68 \mathrm{~mm})$ e 20 mesh $(0,841 \mathrm{~mm})$, tendo sido utilizadas somente as partículas que ficaram retidas na peneira de 20 mesh. Já o gesso fino foi obtido através de comércio local, sem necessidade de pré tratamento (Figura 1).
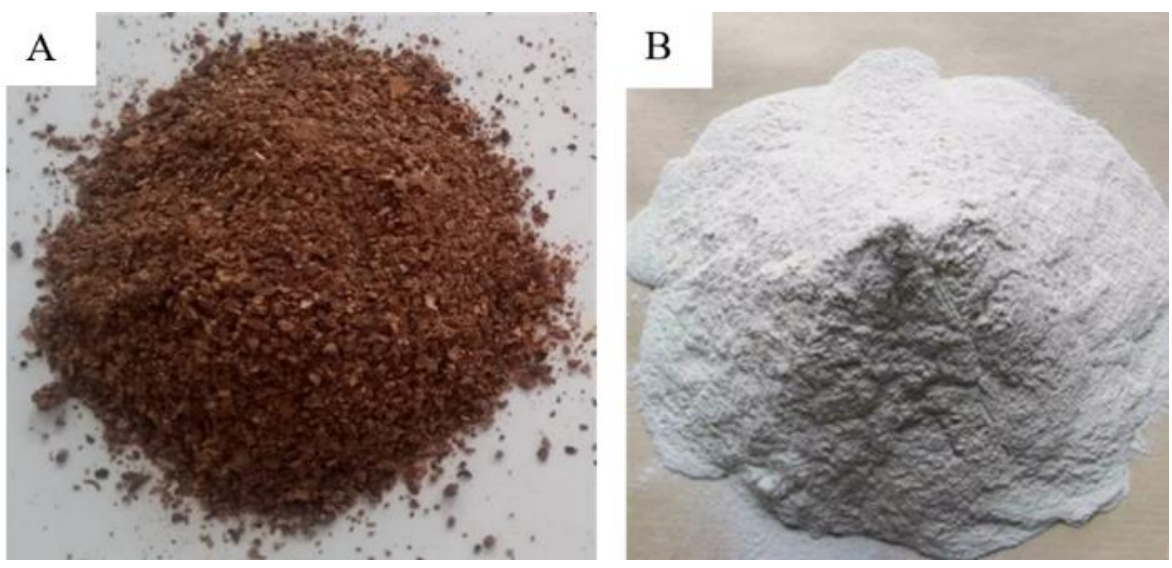

Figura 1: Matéria prima utilizada para produção dos compósitos. A) Resíduo do cacau moído e peneirado e B) gesso fino comercial.

\subsection{Análise do resíduo lignocelulósico}

Para a obtenção da densidade básica do resíduo de cacau, as partículas foram saturadas em água a fim de determinar o volume deslocado (método de imersão). Posteriormente, foram levadas para um forno a $105{ }^{\circ} \mathrm{C}$, onde permaneceram até atingir massa constante, sendo pesada em seguida. A densidade básica, então, foi obtida pela relação entre massa absolutamente seca e volume de massa saturada [14].

Para análise química do resíduo de cacau, as partículas foram moídas em um moinho tipo Willey e peneiradas, sendo utilizadas as que ficaram retidas entre as peneiras de 40 mesh $(0,420 \mathrm{~mm})$ e 60 mesh $(0,250$ $\mathrm{mm})$. Posteriormente foram acondicionadas em ambiente com $65 \pm 3 \%$ de umidade relativa e temperatura de $20 \pm 2{ }^{\circ} \mathrm{C}$. A determinação dos extrativos totais foi realizada com tolueno-etanol e etanol, seguindo a metodologia descrita na NBR 14853 [15]. Para lignina insolúvel, o H2SO4 foi utilizado conforme descrito na NBR 7989 [16], o teor de cinzas foi determinado de acordo com a NBR 13999 [17] utilizando um forno tipo mufla. Já o teor de holocelulose foi determinado pelo método de Browning [18].

Foi realizada ainda, microscopia eletrônica de varredura - MEV do resíduo para fins de caracterização estrutural das partículas. As micrografias foram obtidas por um microscópio eletrônico de varredura JMS $6510\left(\mathrm{JEOL}^{\circledR}\right)$ com uma voltagem de $10 \mathrm{kV}$.

\subsection{Produção dos compósitos}

Foram confeccionados compósitos com a substituição gradual do gesso pelo resíduo do cacau, em relação massa/massa, sendo ao todo cinco composições com seis corpos de prova cada para cada tratamento, totali- 
zando trinta corpos de prova (Tabela 1). A relação água/gesso utilizada para a produção dos compósitos foi de 0,6 sendo escolhida através da observação de uma melhor maleabilidade da mistura com o acréscimo das partículas. Dessa forma, a mesma se tornou homogênea de modo que não fluísse através dos moldes e que sua cura não fosse acelerada.

Tabela 1: Diferentes composições dos compósitos produzidos (relação massa/massa).

\begin{tabular}{ccc}
\hline TRATAMENTO & \multicolumn{2}{c}{ COMPOSIÇÕES } \\
\hline & GESSO (\%) & RESíDUO (\%) \\
\hline T1 & 100 & 0,0 \\
\hline T2 & 97,5 & 2,5 \\
\hline T3 & 95,0 & 5,0 \\
\hline T4 & 92,5 & 7,5 \\
\hline T5 & 90,0 & 10,0 \\
\hline
\end{tabular}

Os corpos de prova foram confeccionados com o auxílio de uma forma feita de MDF, fabricada com fundo em madeira (Figura 2a), de forma a garantir que suas dimensões fossem de 40x40x160 mm, conforme especificações da EN 13279-2 [19]. Após o período de 24 horas, os mesmos foram retirados dos moldes e colocados em local ventilado e livre da ação de intempéries. Durante seis dias os corpos de prova foram reorientados diariamente a fim de garantir uma cura uniforme. No sexto dia, foram pesados e colocados em estufa à $40^{\circ} \mathrm{C}$, até atingir a massa constante. No sétimo dia realizaram-se os ensaios (Figura 2b).
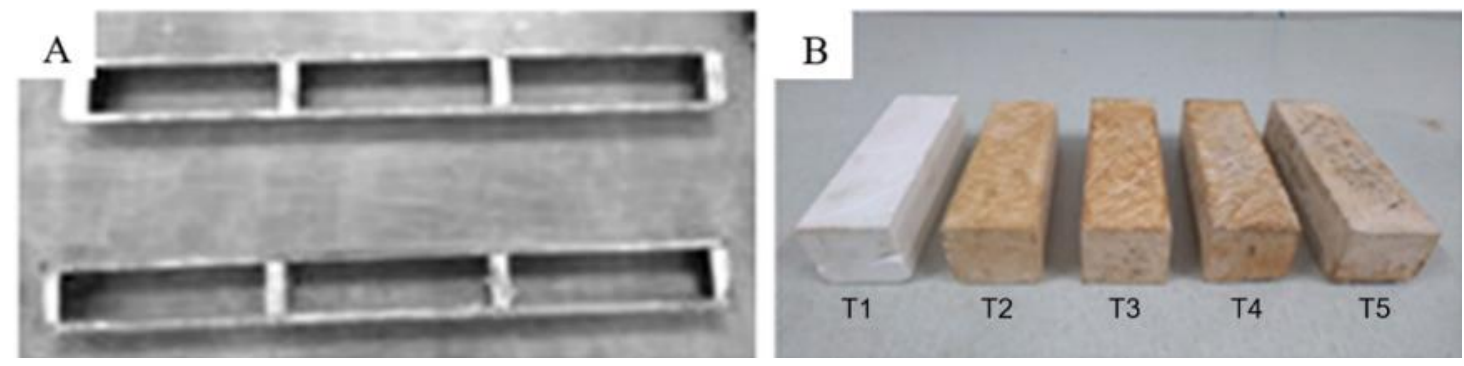

Figura 2: A) Moldes para confecção dos corpos de prova. B) Corpos de prova desmoldados.

\subsection{Avaliação das propriedades físicas e mecânicas}

Para determinação da umidade na base seca foi seguida a NBR 7190 [20] com aferição da massa inicial e final dos corpos de prova. Já a densidade aparente foi determinada pelas diretrizes da NBR 45 [21], a partir da aferição do volume e massa dos corpos de prova. Foi realizado ensaio de absorção de água por capilaridade, em $2 \mathrm{~h}$ de contato, tomando como base as especificações da EN 1015-18 [22].

Em relação às propriedades mecânicas, foi feito ensaio de flexão e compressão em máquina universal de ensaios de acordo com a norma EN 13279-2 [19]. Os corpos de prova para flexão tinham dimensões de 40x40x160 mm, a distância entre os apoios foi de $100 \mathrm{~mm}$ e a carga foi aplicada no centro dos mesmos até a ruptura (Figura 3a). Com esse ensaio foi determinada a resistência de ruptura à flexão (MOR) e a rigidez (MOE) dos corpos de prova. Já para o ensaio de compressão, as dimensões dos corpos de prova foram de 40x40x40 mm sendo, também, a carga aplicada até sua ruptura (Figura 3b). A partir desse ensaio, obteve-se resultados quanto a resistência à compressão $(\mathrm{Rc})$ e a rigidez $(\mathrm{MOE})$ dos compósitos. 

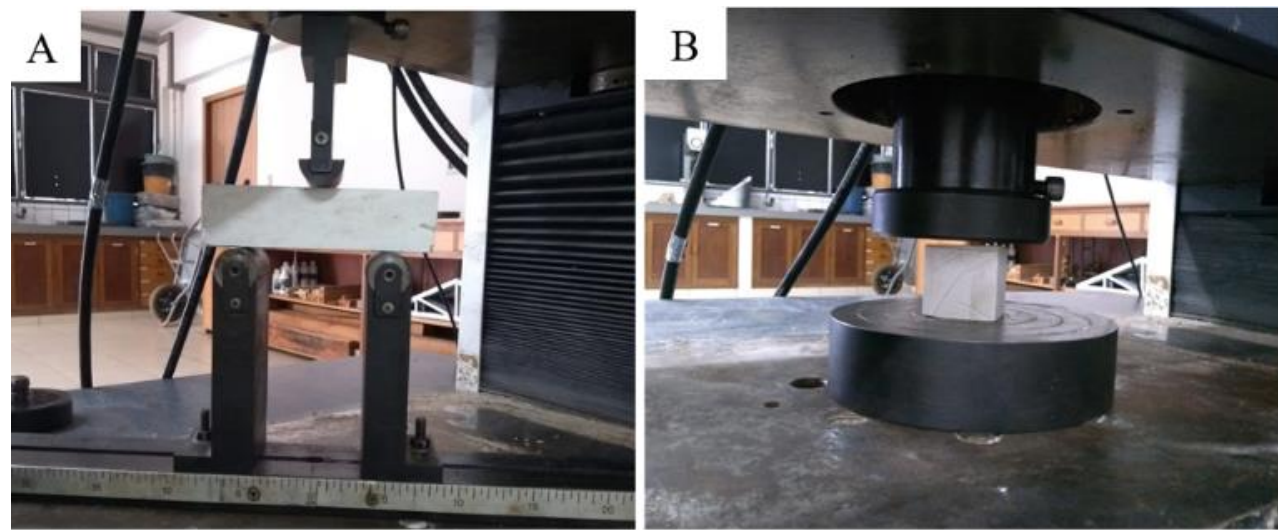

Figura 3: A) Ensaio de flexão e B) ensaio de compressão dos corpos de prova.

\subsection{Avaliação das propriedades acústicas e térmicas}

Para determinação do isolamento acústico, utilizou-se duas câmaras, seguindo a metodologia de TOUTONGE [23]. Os corpos de prova com dimensões de 40x40x160 mm foram posicionados entre as câmaras de emissão (equipada de fonte sonora) e de recepção, separando-as. As medições das intensidades sonoras foram feitas através de um microfone para captação de ruído, nas seguintes frequências: 120, 200, 500, 1000, 2000 e $4000 \mathrm{~Hz}$.

O ensaio para determinação da resistência térmica foi realizado seguindo o mesmo princípio da metodologia de TOUTONGE [23], utilizando duas câmaras, porém dessa vez térmicas. As câmaras eram equipadas com divisória de modo que os corpos de prova de 40x40x160 mm às separassem. A fonte de calor foi posicionada na câmara emissora (equipada de termômetro) e as diferenças de temperaturas foram analisadas com o auxílio de um segundo termômetro posicionado na câmara receptora. O ensaio teve duração de 15 min para cada corpo de prova, obtendo temperatura constante na fonte receptora. As temperaturas foram registradas a cada três minutos nas duas câmaras.

\subsection{Análise dos resultados}

Os resultados das análises físicas, mecânicas e térmicas foram avaliados utilizando delineamento inteiramente casualizado e submetidos à análise de variância e regressão, a 5\% de significância. Já a análise do ensaio acústico foi feita a partir da comparação entre a curva de nível sonoro das câmaras sem divisória e das câmaras com cada compósito testado.

\section{RESULTADOS E DISCUSSÃO}

\subsection{Análise física e química do resíduo}

$\mathrm{O}$ resíduo de cacau apresentou densidade de $0,215 \mathrm{~g} / \mathrm{cm}^{3}$. Este valor é considerado baixo e é característico em resíduos lignocelulósicos como o bagaço de cana-de-açúcar $\left(0,121 \mathrm{~g} / \mathrm{cm}^{3}\right)$ [24] e a vagem de soja $(0,203$ $\mathrm{g} / \mathrm{cm}^{3}$ ) [25]. O fato de os resíduos lignocelulósicos possuírem baixa densidade é interessante para a utilização em compósitos, pois agregam leveza ao produto final já que, geralmente, a densidade da matriz é maior, como no caso do gesso $\left(0,8 \mathrm{~g} / \mathrm{cm}^{3}\right)$ [26].

Em relação às propriedades químicas do resíduo, os resultados estão apresentados na tabela 2. Observase que o teor de extrativos totais foi superior ao encontrado em outros resíduos lignocelulósicos como bagaço de cana-de-açúcar (16,59\%), resíduo da colheita do milho $(17,50 \%)$ e do processamento de café $(8,60 \%)$ [27]. Da mesma forma, o teor de lignina também foi superior a matérias primas como casca de arroz $(20,15 \%)$ e tronco de palma $(19,15 \%)$ [28]. Altos conteúdos desses constituintes nos compósitos podem retardar o endurecimento dos mesmos devido ao abrandamento da cinética de hidratação do gesso e gerar uma incompatibilidade entre matriz e reforço, influenciando as propriedades mecânicas [29, 30].

Para um melhor entendimento dessa influência é explicado que no processo de hidratação do gesso há formação de uma solução supersaturada de íons Ca2+e SO42- que se precipitam em forma de agulhas finas, bem definidas e que entrelaçam entre si, responsáveis pelo bom comportamento mecânico do material [31], [32]. A ação de retardamento da hidratação se deve então, pelo fato de os materiais orgânicos terem grande 
número de grupos -OH e estes interagirem com os íons presentes na fase aquosa [31], [33]. Com isso, ocorre uma mudança na estrutura dos cristais que passam a se apresentar em blocos, sem orientação definida e formatos mais grossos que o normal, ocasionando diminuição das resistências mecânicas devido à baixa aderência entre estes [32].

Tabela 2: Composição química do resíduo do cacau.

\begin{tabular}{cc}
\hline COMPONENTES & TEORES (\%) \\
\hline Extrativos totais & $34,82 \pm 0,13$ \\
\hline Lignina & $35,15 \pm 0,16$ \\
\hline Holocelulose & $28,33 \pm 3,51$ \\
\hline Cinzas & $3,70 \pm 0,63$ \\
\hline
\end{tabular}

Em relação à holocelulose, é observado valor consideravelmente inferior ao encontrado para matérias primas como bagaço de cana-de-açúcar $(71,11 \%)$ [34], casca de arroz (48,39\%) [28] e madeiras em geral (66,08\% e 69,23\% para espécies de eucalipto) [35]. Neste caso, por se tratar de um constituinte higroscópico, a presença de pequenos teores é interessante para evitar uma alta absorção de umidade nos compósitos [36]. Já para o teor de cinzas, o valor encontrado foi menor do que é relatado para outros resíduos como café $(4,92 \%)$, colheita de milho $(6,83 \%)$ e casca de arroz $(16,78 \%)$ [27]. Por se tratar de sílica e outros resíduos minerais, as cinzas auxiliam no retardamento da chama em compósitos de gesso, sendo inerte às outras propriedades [28].

Importante ressaltar que, tratando-se de resíduos lignocelulósicos, os teores dos constituintes químicos sofrem grande variação em relação a espécie, condições de plantio da cultura, condições climáticas, tipo de solo, dentre outros. Dessa forma, explorar as diferenças entre estes serve apenas para fins de comparação com outras matérias primas que têm potencial de serem utilizadas em compósitos. Porém, de modo geral, há uma correlação inversa entre a lignina, a holocelulose e a densidade de forma que quanto maior for o teor de lignina, menor será o teor de holocelulose e, consequentemente, menor será a densidade do material [37]. Essa relação foi claramente observada aqui tendo em vista o baixo teor de holocelulose encontrado no material e sua baixa densidade.

\subsection{Propriedades físicas dos compósitos}

Em relação à densidade aparente, foi observada diferença significativa entre os tratamentos, com decréscimo de valores do tratamento com $100 \%$ de gesso ao tratamento com maior porcentagem de resíduo, de modo que a cada $1 \%$ que este era inserido, foi diminuído aproximadamente 4,87\% dessa propriedade (Figura 4).

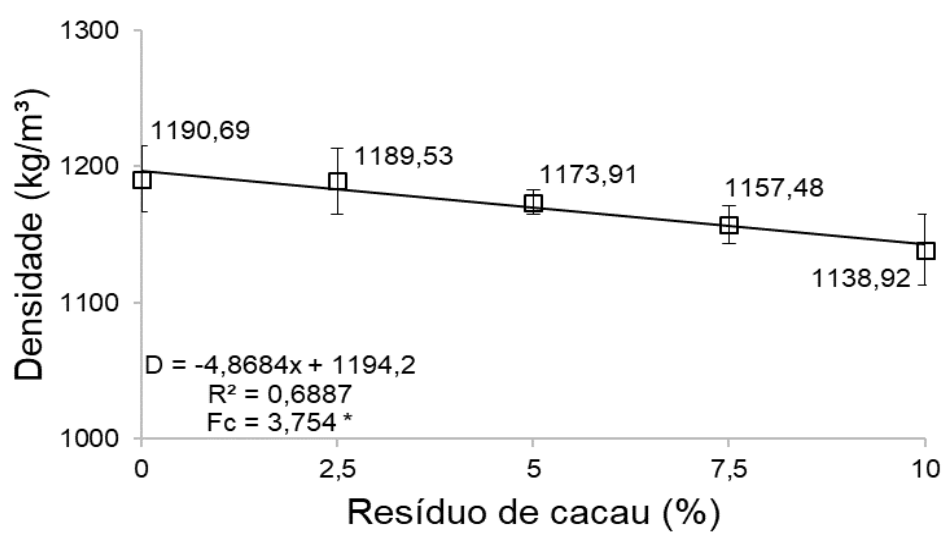

Figura 4: Densidade dos compósitos. * = Relação significativa entre a variável analisada e a porcentagem de resíduo inserido.

Tendo em vista que a densidade do resíduo é menor que a do gesso, a tendência de diminuição dessa propriedade com a inserção do resíduo é esperada. BRAIEK et al. [5] por exemplo, estudando compósitos de gesso reforçados com diferentes porcentagens de fibra de palmeira, concluíram que a densidade diminuía consideravelmente quando aumentava o volume das fibras (de 1.322 para $736 \mathrm{~kg} / \mathrm{m}^{3}$ ). 
Ainda, a densidade de um material pode ser alterada quando é modificada sua massa (em relação ao número de poros) ou seu volume [38]. Ao incluir materiais lignocelulósicos em uma matriz de gesso, há um aumento na porosidade de modo que quanto maior o número de partículas inseridas, menor será a densidade do compósito e maior absorção de água [29]. Ainda, ressalta-se que o tegumento da amêndoa do cacau tem como característica um alto grau de porosidade [39], o que pode influenciar na sua baixa densidade e contribuir ainda mais para o aumento da porosidade do compósito.

$\mathrm{Na}$ imagem de microscopia eletrônica de varredura - MEV pode ser observada a estrutura porosa do resíduo (Figura 5). Pode-se dizer então que houve aumento de vazios nos compósitos, devido a característica intrínseca do resíduo e provavelmente nas interfaces reforço-matriz, tornando-os mais leves. A baixa densidade dos materiais lignocelulósicos é uma das justificativas de se fazer a inclusão na matriz de gesso [4] já que facilita a utilização, principalmente na construção civil para instalações de chapas e revestimentos.

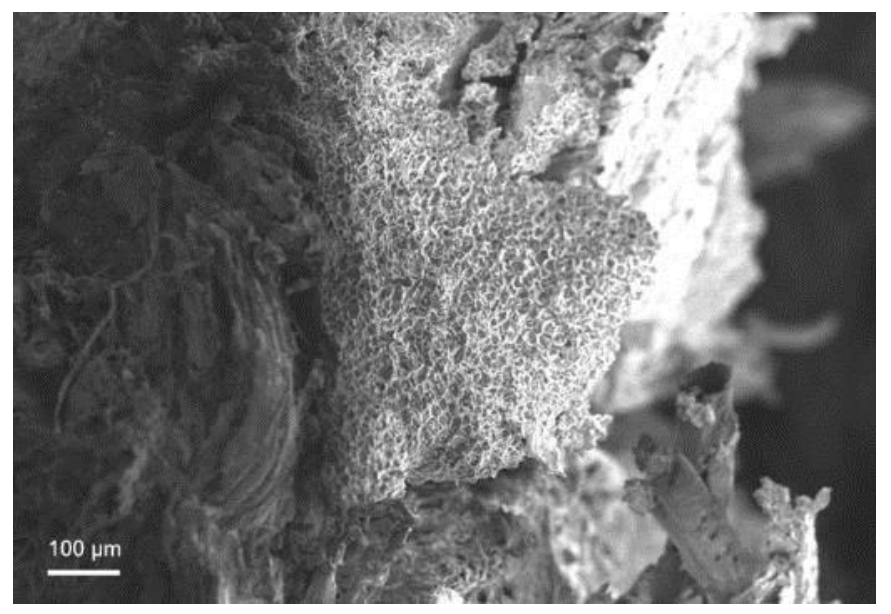

Figura 5: Imagem de microscopia eletrônica de varredura do resíduo de cacau.

Para a umidade, o ajuste da regressão quadrática foi significativo $(\mathrm{Fc}=7,318)$. Isso significa que houve diferença estatística significativa entre os tratamentos com relação decrescente dessa propriedade, à medida em que se acresce resíduo de cacau nos compósitos (Figura 6).

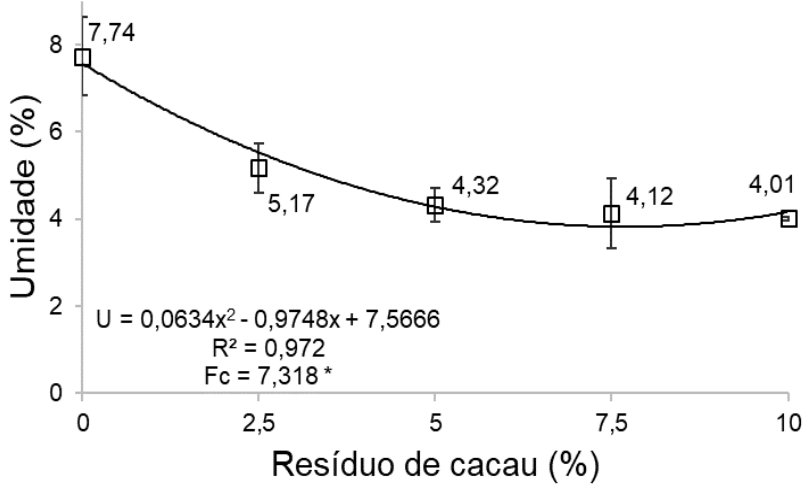

Figura 6: Umidade dos compósitos. * = Relação significativa entre a variável analisada e a porcentagem de resíduo inserido.

Têm-se encontrado na literatura, compósitos reforçados com materiais lignocelulósicos em que a umidade é aumentada com a inserção dos mesmos. CHINTA et al. [40] por exemplo, obtiveram valores de 0,48 a 1,46\% para compósitos de gesso com fibras de coco, juta, lã, algodão e banana. Essa diferença de tendência é explicada pelas propriedades químicas do material.

O conjunto holocelulose (celulose e hemicelulose), por ter caráter higroscópico, faz com que os materiais que tenham alto teor destes constituintes sejam mais propensos a adsorverem umidade, o que foi apontado pelos autores e que é normalmente encontrado nos trabalhos. O contrário ocorreu aqui pois os consideráveis teores de lignina e extrativos no resíduo de cacau (aproximadamente 70\%), que são hidrofóbicos, podem ter 
inibido essa propriedade. Confirmando isso, é relatado que o aumento de extrativos no material lignocelulósico tem influência direta com a diminuição da umidade [41].

Para a absorção de água, o ajuste da regressão foi significativo $(\mathrm{Fc}=18,303)$, indicando diferença estatística entre tratamentos com relação quadrática decrescente a medida em que se acresce resíduo de cacau (Figura 7).

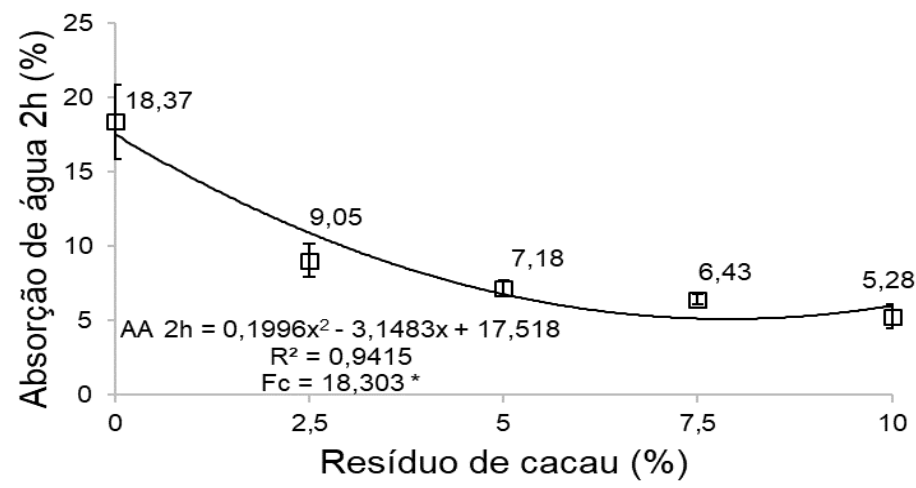

Figura 7: Absorção de água dos compósitos. * = Relação significativa entre a variável analisada e a porcentagem de resíduo inserido.

Assim como para a umidade, é encontrada na literatura a tendência de aumento da absorção de água em compósitos com o acréscimo de materiais lignocelulósicos devido suas características hidrofílicas [38, 40], [42]. Porém, como foi visto na análise química, o resíduo do cacau apresentou teores de constituintes hidrofóbicos, como a lignina, consideravelmente superiores a outros resíduos. Estes conferem impermeabilidade ao material [43]. Dessa forma, a diminuição da absorção de água nos compósitos pode ser explicada seguindo a mesma lógica aplicada à umidade e, pode-se dizer que o aumento desses constituintes hidrofóbicos no compósito causou uma maior dificuldade do mesmo em absorver água após sua cura.

Uma das maiores deficiências do gesso como material de construção é sua suscetibilidade à água [1], o que restringe seu uso à ambientes secos. $\mathrm{O}$ aumento da hidrofobicidade com a inserção do resíduo se mostra benéfica no sentido de reduzir tal restrição e ampliar a utilização do gesso quando compósito. Ainda, com o intuito de diminuir os fenômenos de degradação dos materiais pretende-se que os corpos de prova tenham menores coeficientes de absorção e que esse fenômeno ocorra de forma lenta [38].

\subsection{Propriedades mecânicas dos compósitos}

No ensaio de flexão estática, os resultados encontrados para a resistência de ruptura à flexão (MOR) (Figura 8a) e módulo de elasticidade (MOE) (Figura 8b) mostram que os compósitos sem adição de resíduo apresentaram maiores resistências e que estas foram diminuídas gradativamente ao longo dos demais tratamentos havendo diferença estatística significativa entre estes. A cada $1 \%$ de resíduo inserido, houve redução de 0,2706 MPa para MOR e de 197,09 MPa para MOE.

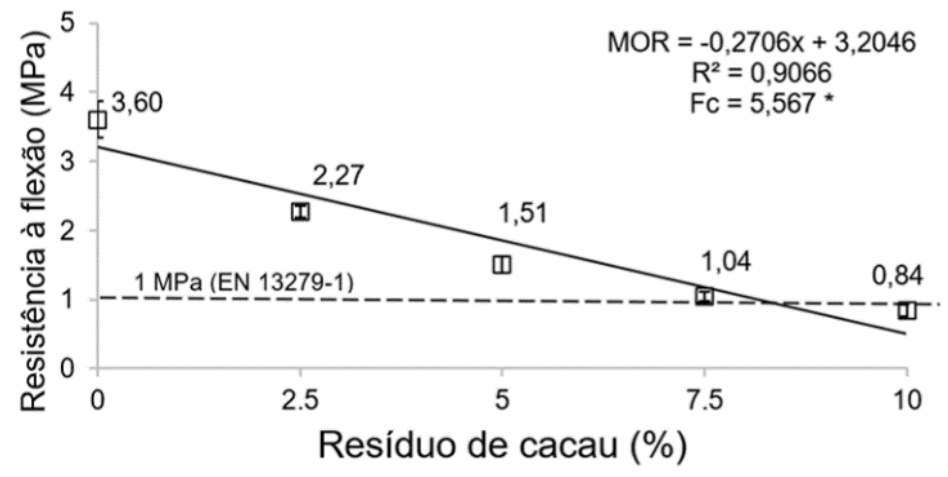




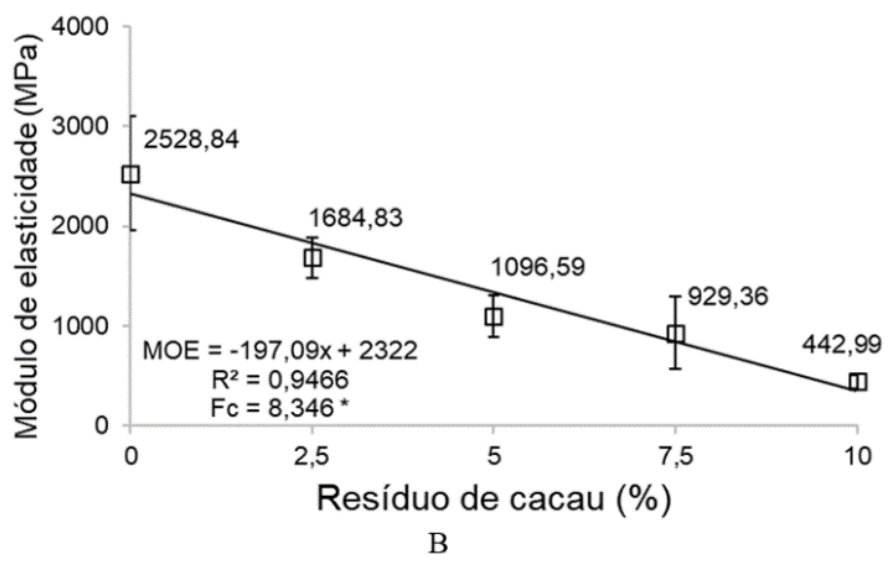

Figura 8: (A) Resistência de ruptura à flexão e (B) módulo de elasticidade dos compósitos. * = Relação significativa entre a variável analisada e a porcentagem de resíduo inserido.

A norma EN 13279-1 [44], preconiza valor de 1 MPa para a resistência de ruptura à flexão. Dessa forma, observando a tendência de redução na figura $8 \mathrm{a}$, a inclusão de até $8,15 \%$ de resíduo atende o requerido pela normatização.

Em teoria, compósitos contendo fibras são macios e dúcteis em torno das fibras (que são geralmente mais fortes) mas podem ser frágeis em relação à matriz [45]. De acordo com essa regra, cada fase constituinte do compósito contribui para suas propriedades, sendo essa função dependente apenas da fração volumétrica dos constituintes, porém, essa teoria não considera a presença de vazios nem os efeitos da interface entre a matriz e a carga [46].

Para testes de flexão em compósitos, a interação entre matriz e reforço é de grande importância pois espera-se que haja uma transmissão uniforme de cargas entre os constituintes. A exemplo disso, IUCOLANO et al. [6] estudando compósitos de gesso com fibras de abacá, obtiveram baixos valores de resistência à flexão e remetem a uma fraca adesão entre as fibras brutas e o gesso. Já ao realizarem tratamento prévio das fibras, maiores valores foram encontrados pois houve aumento do intertravamento fibra-matriz. Dessa forma, além do aumento da porosidade nos compósitos, os constituintes químicos do resíduo do cacau, como a lignina e os extrativos, podem ser indicados como causadores da fraca interação entre as fases [32, 33].

ANTUNES et al. [47] também observaram diminuição de valores na resistência à flexão em seus compósitos a medida em que aumentavam o teor de casca de arroz. É relatado na literatura que pode haver um teor ideal de fibra, variando de acordo com o seu tipo e granulometria para que a resistência mecânica seja otimizada e, além disso, a formulação do compósito e a dispersão não uniforme das fibras na matriz também estão relacionadas com a diminuição das resistências à flexão [46, 47].

Em relação ao ensaio de compressão, os resultados mostram diferença significativa entre os tratamentos tanto para a resistência à compressão (Figura 9a) quanto para o módulo de elasticidade (Figura 9b), com diminuição progressiva das resistências dos compósitos com o acréscimo de resíduo. A diminuição observada a cada $1 \%$ de resíduo inserido foi de 0,296 MPa para resistência à compressão e de 109,43 MPa para módulo de elasticidade. 


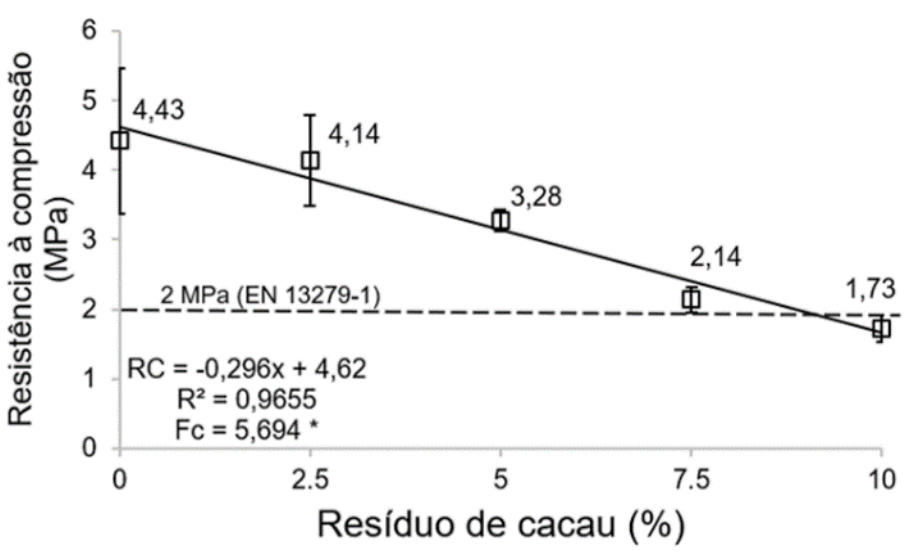

A

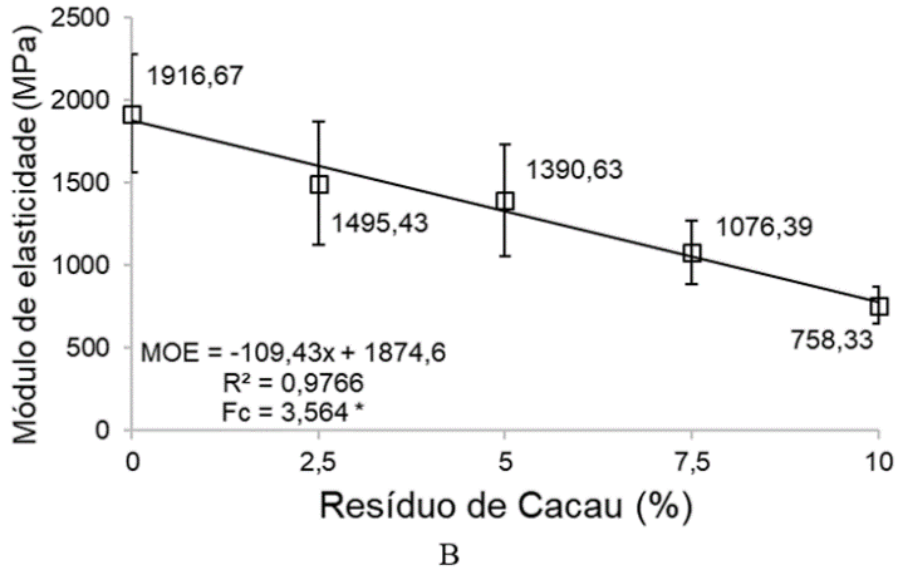

Figura 9: A) Resistência à compressão e B) módulo de elasticidade dos compósitos. * = Relação significativa entre a variável analisada e a porcentagem de resíduo inserido.

Com base nas diretrizes da EN 13279-1 [44], o valor mínimo exigido para a resistência à compressão é de $2 \mathrm{MPa}$. Assim calcula-se que a inclusão de até $8,85 \%$ de resíduo de cacau na matriz de gesso atende o preconizado pela norma (Figura 9a).

Essa redução de valores já era esperada tendo em vista que, de modo geral, quanto maior a quantidade de materiais lignocelulósicos inseridos na matriz de gesso, menor tende a ser a resistência à compressão [4]. Isso se deve à estrutura menos compacta dos resíduos em relação ao gesso, o que proporciona uma menor compactação na estrutura do compósito. Esse fenômeno pode resultar em uma matriz insuficiente para cobrir as partículas e constrói sistemas de rede dentro dos compósitos e, portanto, menos eficiência na transferência de carga [42].

Resultados parecidos são encontrados na literatura e à estes são atribuídos fatores como a porosidade do material e do compósito, microfissuras presentes no compósito, o formato e tamanho das partículas, que podem provocar heterogeneidade e segregação em áreas do compósito, e a fraca interface entre o resíduo e o gesso [29, 42, 48, 49]. É relatado também que o aumento do teor de água nos compósitos tem implicação direta com a diminuição das resistências mecânicas devido a sua infiltração entre os cristais do gesso, enfraquecendo sua estrutura [29, 50]. Porém, essa regra não foi observada aqui já que os teores de umidade e absorção de água reduziram juntamente com as resistências. O "enfraquecimento dos compósitos", então, não está relacionado à ação da água em suas estruturas.

Visto isso, entende-se que os fatores que mais impactaram na diminuição de valores das propriedades mecânicas, tanto na flexão quanto na compressão, foram a estrutura porosa e menos compacta do resíduo, a criação de poros no compósito e a fraca interação entre matriz e reforço devido aos constituintes químicos do material. Em suma, pode-se dizer que os consideráveis teores de lignina e extrativos totais do resíduo de cacau foram os principais influenciadores tanto das propriedades físicas, por serem hidrofóbicos, quanto das propriedades mecânicas, pela influência causada na cinética de hidratação do gesso. 


\subsection{Resistência térmica dos compósitos}

A resistência térmica de um material mede a sua capacidade de reduzir a troca de calor entre ambientes. Assim, quanto maior for essa resistência do material, melhor é a sua capacidade de isolação térmica [51]. A partir da análise feita nos compósitos, foi observada tendência de acréscimo de valores com diferença significativa entre os tratamentos (Figura 10). A relação de aumento encontrada foi de $0,0466 \mathrm{~K} * \mathrm{~m} / \mathrm{W}$ para cada $1 \%$ de resíduo que era inserido.

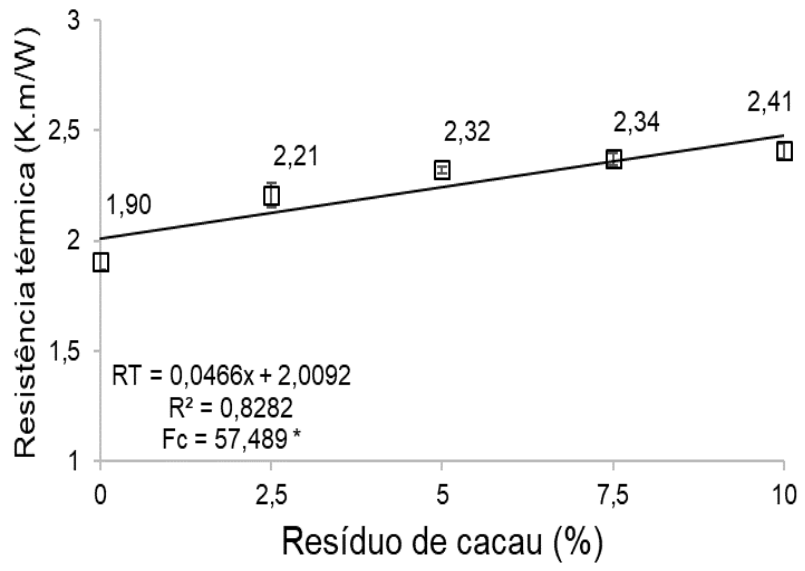

Figura 10: Resistência térmica dos compósitos. * = Relação significativa entre a variável analisada e a porcentagem de resíduo inserido.

O aumento observado pode ser explicado pelo fato de os materiais lignocelulósicos apresentarem coeficientes de condutibilidade inferiores ao do gesso, já que essa é inversamente proporcional à resistência térmica. A condutividade térmica do gesso é de $0,5 \mathrm{~W} / \mathrm{m} . \mathrm{K}$, enquanto a de materiais lignocelulósicos varia de 0,12 a $0,29 \mathrm{~W} / \mathrm{m} . \mathrm{K}$ [52]. Dessa forma, pode-se dizer também que a condutibilidade térmica dos compósitos foi reduzida com a inserção do resíduo.

Essa tendência de aumento da resistência térmica também foi relatada na literatura para compósitos de gesso com hastes de girassol e resíduos têxteis [53], fibras de palma [5], hastes de girassol trituradas e casca de arroz [54], aparas de madeira [55], dentre outros. Os resultados são atribuídos não só à baixa condutividade térmica dos resíduos, mas também à densidade dos compósitos. Uma menor densidade, ou seja, maior número de vazios, faz com que compósitos tendam a ter condutividades térmicas mais baixas devido à baixa propagação do calor no espaço [47]. Isso pode ser relacionado aqui, ao observar a característica porosa do resíduo e o aumento da porosidade do compósito ao longo dos tratamentos.

\subsection{Isolamento acústico dos compósitos}

Quanto ao isolamento acústico, em termos de comparação com as câmaras sem divisória, nota-se que os compósitos reduziram o ruído em todas as frequências analisadas (Figura 11). A capacidade de um material permitir ou não absorção sonora está relacionada com sua porosidade. A típica tendência de materiais porosos expostos ao som é de ocorrer vibração das moléculas de ar dentro dos poros, transformando energia em calor [56]. 


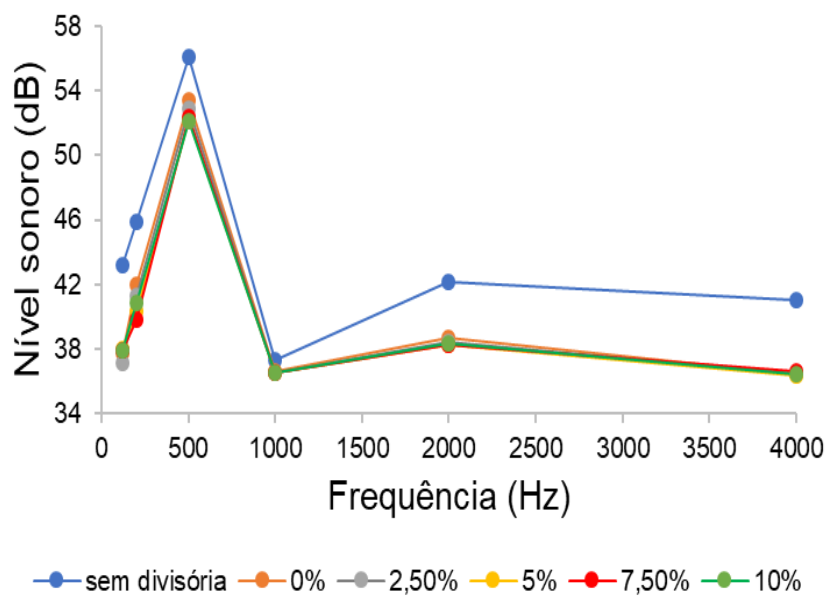

Figura 11: Isolamento acústico dos compósitos.

Dessa forma, a melhoria na absorção sonora de compósitos a base de gesso e resíduos lignocelulósicos é relatada na literatura como resposta ao aumento do número de poros devido a interação do reforço com a matriz, que deixa vazios nas interfaces e/ou cavidades interconectadas [56, 57]. Ainda, observa-se que as maiores reduções ocorreram a partir de $2000 \mathrm{~Hz}$ e isso pode ser explicado pelo fato de que, em baixas frequências as perdas de energia são isotérmicas e, portanto, limitadas, enquanto que nas altas frequências são adiabáticas e geralmente mais significativas.

Observando a figura 11, não se percebe diferença considerável entre tratamentos. Porém, os valores encontrados indicam diminuição de ruído na faixa de 0,78 a 2,43\% em todas as frequências com a inclusão do resíduo. Essas pequenas reduções demonstram a influência da porosidade no interior dos compósitos ocasionada pelo resíduo e sua interação com a matriz, mesmo que sem linearidade. Isso indica que, independente da porcentagem de resíduo inserido, houve melhoria na capacidade de absorção sonora em todas as frequências analisadas.

\section{CONCLUSÕES}

Os reforços adicionados aos compósitos resultaram em um produto final mais leve, o que é atrativo em muitos usos como na construção civil pois possibilitará diminuição dos esforços nas vigas, pilares e fundações. Da mesma forma, a umidade e absorção de água dos compósitos diminuíram com a inclusão do resíduo. Esse resultado também se mostra benéfico tendo em vista o alto caráter hidrofílico do gesso puro e que por vezes restringe seu uso a ambientes secos.

Para as propriedades mecânicas houve diminuição de resistências influenciada principalmente pelas características químicas e físicas intrínsecas do resíduo e a possível incompatibilidade entre as fases. Nesse sentido, o resíduo demostrou potencialidade para inclusão de até $8,15 \%$ em massa na matriz de gesso, visto que este foi o valor que atendeu a normatização em todas as propriedades analisadas.

A resistência térmica dos compósitos foi melhorada, o que traz vantagens na sua utilização, por exemplo, em placas de gesso para vedação ou forro, pois garantirá maior conforto térmico para a edificação. Da mesma forma, o isolamento acústico apresentou melhorias nos compósitos em relação ao gesso puro, principalmente para isolamento de sons agudos, embora não tenha havido linearidade entre os tratamentos.

Este estudo visou verificar a viabilidade de se produzir um compósito de matriz de gesso com a inserção de um resíduo agrícola em sua composição. Os resultados se mostraram satisfatórios tendo em vista a possibilidade de valorizar um subproduto lignocelulósico e também de criar um material inovador com alto valor agregado.

\section{PERSPECTIVAS FUTURAS}

O resíduo do cacau apresentou potencialidades para produção do compósito a base de gesso, no entanto, alguns fatores relacionados ao processo necessitam ser melhor explorados. Assim, como perspectivas para próximos estudos, os parâmetros de produção dos compósitos podem ser melhor ajustados na tentativa de viabilizar a inclusão do resíduo sem causar prejuízo às propriedades do material e até mesmo possibilitar uma maior porcentagem de inserção. 
Além disso, análises mais aprofundadas das propriedades físicas, químicas do resíduo são necessárias para um melhor entendimento da interação deste com a matriz de gesso. Ressalta-se ainda que um maior conhecimento sobre essa matéria prima subsidiará sua utilização em novos trabalhos e para a produção de outros materiais inovadores como adsorventes para águas residuais [58, 59], materiais coagulantes sustentáveis [60], nanocompósitos para baterias [61], dentre outros.

\section{AGRADECIMENTOS}

Os autores agradecem a Universidade Federal de Lavras - UFLA, Coordenação de Aperfeiçoamento de Pessoa de Nível Superior - CAPES, Conselho Nacional de Desenvolvimento Científico e Tecnológico - CNPq e a Fundação de Amparo à Pesquisa do Estado de Minas Gerais - FAPEMIG, que colaboraram para a realização deste estudo.

\section{BIBLIOGRAFIA}

[1] PINTO, N.A., FIORITI, C.F., BERNABEU, J.P., et al., "Avaliação de matriz de gesso com incorporação de borracha de pneus para utilização na construção civil," Revista Tecnológica, v. 25, n. 1, pp. 103-117, 2016.

[2] United Nations Organization, ONU, “Transforming Our World: The 2030 Agenda for Sustainable Development," https://sustainabledevelopment.un.org/post2015/transformingourworld., acessado em março de 2020.

[3] CALLISTER JUNIOR, D.G., RETHWISCH, W.D., Ciência e engenharia de materiais: uma introdução. 9 ed. São Paulo, LTC, 2016.

[4] MORALES-CONDE, M.J., RODRÍGUEZ-LIÑÁN, C., PEDREÑO-ROJAS, M.A., "Physical and mechanical properties of wood-gypsum composites from demolition material in rehabilitation works," Construction and Building Materials, v. 114, n. 2016, pp. 6-14, 2016.

[5] BRAIEK, A., KARKRI, M., ADILI, A., et al., "Estimation of the thermophysical properties of date palm fibers/gypsum composite for use as insulating materials in building," Energy and Buildings, v. 140, pp. 268279, 2017.

[6] IUCOLANO, F., CAPUTO, D., LEBOFFE, F. et al., "Mechanical behavior of plaster reinforced with abaca fibers," Construction and Building Materials, v. 99, pp. 184-191, 2015.

[7] SILVA, L., "Material compósito à base de gesso reforçado com fibras: caracterização mecânica,"Engenharia Civil, n. 49, pp. 5-14, 2014.

[8] Instituto Brasileiro de Geografia e Estatísticas, IBGE, "Levantamento sistemático da produção agrícola," https://www.ibge.gov.br/estatisticas/economicas/agricultura-e-pecuaria/9201-levantamento-sistematico-daproducao-agricola.html, acessado em julho de 2020.

[9] SILVA, R.B., FONTES, C.M.A., LIMA, P.R.L., et al., "Biomass ash from cocoa agroindustry: characterization and use as a cement substitute," Ambiente Construído, v. 15, n. 4, pp. 321-334, Dec. 2015.

[10] VÁSQUEZ, Z.S. et al., "Biotechnological approaches for cocoa waste management: A review," Waste Management, v. 90, pp. 72-83, 2019.

[11] ARLORIO, M., COISSON, J.D., RESTANI, P., et al., "Characterization of Pectins and Some Secondary Compounds from Theobroma cacao Hulls,” Journal of Food Science, v. 66, n. 5, pp. 653-656, Jun. 2001.

[12] BADIYANI, B.K., KUMAR, A., BHAT, P.K. et al., "Chocolate Disinfectant: Effectiveness of Cocoa Bean Husk Extract on Streptococcus mutans in Used Toothbrushes," International Journal of Ophthalmology and Clinical Research, v. 1, n. 1, pp. 7-10, 2013.

[13] FIORESI, F., et al., "Chemical modification of the cocoa shell surface using diazonium salts," Journal of Colloid and Interface Science., v. 494, pp. 92-97, May 2017.

[14] MARTINS, E.H., VILELA, A.P., MENDES, R.F., et al., "Soybean waste in particleboard production," Ciência e Agrotecnologia, v. 42, n. 2, pp. 186-194, Mar. 2018.

[15] ASSOCIAÇÃO BRASILEIRA TÉCNICA DE CELULOSE E PAPEL, ABNT, NBR 14853, Determinação do material solúvel em etanol-tolueno e em diclorometano e em acetona, Rio de Janeiro, 2010.

[16] ASSOCIAÇÃO BRASILEIRA TÉCNICA DE CELULOSE E PAPEL, ABNT, NBR 7989, Pulp and wood - Determination of acid-insoluble lignina, Rio de Janeiro, 2010.

[17] ASSOCIAÇÃO BRASILEIRA TÉCNICA DE CELULOSE E PAPEL, ABNT, NBR 13999, Papel, tábua, polpas e madeira - determinação de resíduo (cinzas) na ignição a $525{ }^{\circ} \mathrm{C}$, Rio de janeiro, 2003. 
[18] BROWNING, B.L., The chemistry of wood, New York, Interscience, p. 689, Apr. 1964.

[19] EUROPEAN COMITTE OF STANDARDIZATION, CEN, EN 13279-2, Gypsum binders and gypsum plasters; Part 2: Test methods, Bruxelas, 2006.

[20] ASSOCIAÇÃO BRASILEIRA DE NORMAS TÉCNICAS, ABNT, NBR 7190, Projetos de estruturas de madeira, Rio de janeiro, 1997.

[21] ASSOCIAÇÃO BRASILEIRA DE NORMAS TÉCNICAS, ABNT, NBR NM 45, Agregados - Determinação da massa unitária e do volume de vazios, Rio de Janeiro, 2006.

[22] EUROPEAN COMMITTEE FOR STANDARDIZATION, CEN, EN 1015-18, Methods of test for mortar for masonry - Part 18: Determination of water absorption coefficient due to capillary action of hardened mortar, Brussels, 2002.

[23] TOUTONGE, J.A. "Projeto e construção de câmaras reverberantes em escala reduzida para o estudo das características de perda de transmissão de divisórias confeccionadas a partir de materiais regionais", Tese de M.Sc., Universidade Federal do Pará, Belém, PA, Brasil, 2006.

[24] SOARES, S.S., GUIMARÃES JÚNIOR, J.B., MENDES, L.M. et al., "Valorization of sugarcane bagasse for production of low density particleboards," Revista Ciência da Madeira, v. 8, n. 2, pp. 64-73, May 2017.

[25] LISBOA, F.J.N., SCATOLINO, M.V., PROTÁSIO, T.P. et al., "Lignocellulosic Materials for Production of Cement Composites: Valorization of the Alkali Treated Soybean Pod and Eucalyptus Wood Particles to Obtain Higher Value-Added Products," Waste and Biomass Valorization, v. 11, n. 5, pp. 2235-2245, 2018.

[26] ASSOCIAÇÃO BRASILEIRA DE NORMAS TÉCNICAS, ABNT, NBR 13207, Gesso para construção civil: especificações, Rio de Janeiro, 1994.

[27] PROTÁSIO, T.P., TONOLI, G.H.D., GUIMARÃES, M., et al., "Correlações canônicas entre as caractrísticas químicas e energéticas de resíduos lignocelulósicos," Cerne, v. 18, n. 3, pp. 433-439, 2012.

[28] SELAMAT, M.E., HASHIM, R., SULAIMAN, O., et al., "Comparative study of oil palm trunk and rice husk as fillers in gypsum composite for building material," Construction and Building Materials, v. 197, pp. 526-532, 2019.

[29] SHIROMA, L., CAMARINI, G., BERALDO, A.L., "Effect of wood particle treatment on the properties of gypsum plaster pastes and composites," Revista Matéria, v. 21, n. 4, pp. 1032-1044, Oct. 2016.

[30] WEBER, A.M., CECHIN, L., TOKARSKI, R.B. "Análise da influência do traço nas propriedades do compósito cimento-madeira," Revista Principia, v. 1, n. 36, pp. 50-59, 2017.

[31] MENEZES, R., POVOAS, Y. "Influência de aditivo retardador de pega na reciclagem da pasta de gesso utilizada como revestimento interno de parede," Revista de Engenharia e Pesquisa Aplicada, v. 2, n. 1, Nov. 2016.

[32] HINCAPIE, A.M., CINCOTTO, M.A., "Efeito de retardadores de pega no mecanismo de hidratação e na microestrutura do gesso de construção," Ambiente Construído, v. 1, n. 2, pp. 07-16, 1997.

[33] CASAGRANDE, C.A., JOCHEM, L.F., ONGHERO, L., et al., "Effect of partial substitution of superplasticizer by silanes in Portland cement pastes," Journal of Building Engineering, vol. 29, p. 101226, May 2020.

[34] PROTÁSIO, T.P., MENDES, R.F., SCATOLINO, M.V., et al., "Thermal stability of particleboards of sugar cane bagasse and Pinus spp.," Scientia Forestalis, v. 43, n. 107, pp. 683-691, 2015.

[35] BRAZ, R.L. et al., "Caracterização anatômica, física e química da madeira de clones de Eucalyptus cultivados em áreas sujeitas à ação de ventos," Ciência da Madeira, v. 5, pp. 127-137, 2014.

[36] GUIMARÃES JÚNIOR, J.B., XAVIER, M.M., SANTOS, T.S., et al., "Addition of sorghum culture waste in eucalyptus particleboards," Pesquisa Florestal Brasileira, v. 36, n. 88, p. 435, Jan. 2017.

[37] TRUGILHO, P.F., LIMA, J.T., MENDES, L.M., "Influência da idade nas características físico-químicas e anatômicas da madeira de Eucalyptus saligna," Cerne, v. 2, n. 1, pp. 1-15, 1996.

[38] RIBEIRO, J.S., SERRA, J.C.V., "Compósitos Obtidos de Resíduos de Papel, Reforçados com Pó de Serragem e de Gesso," Engineering and Science, v. 2, n. 1, pp. 1-9, 2014.

[39] ADEGOKE, K.A., BELLO, O.S., "Dye sequestration using agricultural wastes as adsorbents," Water Resources and Industry, v. 12, pp. 8-24, Dec. 2015.

[40] CHINTA, S.K., KATKAR, P.M., JAFER, M.M. "Natural fibres reinforced gypsum composites," International Journal of Management Science and Engineering, v. 4, n. 3, pp. 318-325, 2013.

[41] JANKOWSKY, I.P. GALVÃO, A.P.M. "Influência do teor de extrativos na umidade de equilíbrio da 
madeira," Instituto de Pesquisas e Estudos Florestais, n. 18, pp. 1-33, 1979.

[42] DAI, D., FAN, M., "Preparation of bio-composite from wood sawdust and gypsum," Industrial Crops and Products, v. 74, pp. 417-424, Nov. 2015.

[43] BHATIA, L., JOHRI, S., AHMAD, R., "An economic and ecological perspective of ethanol production from renewable agro waste: a review," AMB Express, v. 2, n. 1, p. 65, 2012.

[44] EUROPEAN COMMITTEE FOR STANDARDIZATION, CEN, EN 13279-1, Gesso e produtos à base de gesso para a construção; Parte 1: Definições e requisitos, Bruxelas, 2008.

[45] SMITH, E.H. Mechanical Engineer's Reference Book, Butterwort. 1994.

[46] ROCHA, T.M.S., MIRANDA, L.F.R., PARCHEN, C.F.A., "Influência da composição granulométrica das partículas de resíduos de madeira nas propriedades de compósitos minerais: parte 2: gesso-madeira," Ambiente Construído, v. 19, n. 4, pp. 195-206, 2019.

[47] ANTUNES, A., FARIA, P., SILVA, V. et al., "Rice husk-earth based composites: A novel bio-based panel for buildings refurbishment," Construction and Building Materials, v. 221, pp. 99-108, 2019.

[48] KHALIL, A.A., TAWFIK, A., HEGAZY, A.A., et al., "Effect of some waste additives on the physical and mechanical properties of gypsum plaster composites," Construction and Building Materials, v. 68, pp. 580-586, 2014.

[49] MARWARDT, L.J., WILSON, T.R.C., "Strength and related properties of woods grown in the United States. Washington: USDA. (Technical Bulletin, 479),." pp. 67, R.C., St, 1935.

[50] COQUARD, P., BOISTELLE, R., "Hardness, elasticity modulus and flexion strength of dry set plaster," Journal of Materials Science, v. 29, n. 17, 1994.

[51] ADAMY, A.P.A., ROSA, F.S., ROSA, L.C., "Desempenho térmico de compósitos à base de talos de girassol em componentes de edificações," Espacios, v. 37, n. 15, 2016.

[52] ASSOCIAÇÃO BRASILEIRA DE NORMAS TÉCNICAS, ABNT, NBR 15220, Desempenho térmico de edificações Parte 2: Métodos de cálculo da transmitância térmica, da capacidade térmica, do atraso térmico e do fator solar de elementos e componentes de edificações. Rio de Janeiro, 2005.

[53] BINICI, H., EKEN, M., DOLAZ, M., et al., "An environmentally friendly thermal insulation material from sunflower stalk, textile waste and stubble fibres," Construction and Building Materials, v. 51, pp. 24-33, 2014.

[54] KOCAMAN, I., SISMAN, C.B., GEZER, E., "Investigation the using possibilities of some mineralbound organic composites as thermal insulation material in rural buildings," Scientific Research and Essays, v. 6, n. 7, pp. 1673-1680, 2011.

[55] ADILI, A., LACHHEB, M., BRAYEK, A., et al., "Estimation of thermophysical properties of lightweight mortars made of wood shavings and expanded polystyrene beads using a hybrid algorithm," Energy and Buildings, v. 118, pp. 133-141, Apr. 2016.

[56] MORETTI, E., BELLONI, E., AGOSTI, F., "Innovative mineral fiber insulation panels for buildings: Thermal and acoustic characterization," Applied Energy, v. 169, pp. 421-432, 2016.

[57] LAHOUIOUI, M., BEN ARFI, R., FOIS, M. et al., "Investigation of Fiber Surface Treatment Effect on Thermal, Mechanical and Acoustical Properties of Date Palm Fiber-Reinforced Cementitious Composites," Waste and Biomass Valorization, v. 11, n. 8, pp. 4441-4455, 2019.

[58] CHEN, J., WANG, X., HUANG, Y., et al., "Adsorption Removal of Pollutant Dyes in Wastewater by Nitrogen-doped Porous Carbons Derived from Natural Leaves," Engineered Science, v. 5, pp. 30-38, 2018.

[59] WEI, H., MA, J., SHI, Y., et al., "Sustainable Cross-linked Porous Corn Starch Adsorbents with High Methyl Violet Adsorption," ES Materials \& Manufacturing, v. 2, pp. 28-34, 2018.

[60] ZHAO, S., SUN, Q., GU, Y., et al., "Enteromorpha prolifera polysaccharide based coagulant aid for humic acids removal and ultrafiltration membrane fouling control," International Journal of Biological Macromolecules, v. 152, pp. 576-583, Jun. 2020.

[61] BATOOL, S., IDREES, M., KONG, J., et al., "Assessment of the electrochemical behaviour of silicon@carbon nanocomposite anode for lithium-ion batteries," Journal of Alloys and Compounds, v. 832, p. 154644, Aug. 2020. 
ORCID

Maria Cecillia Ramos de Araújo Veloso

Luciana Silva Villela

Laércio Mesquita Júnior

José Benedito Guimarães Júnior

Mara Lúcia Agostini Valle

Lourival Marin Mendes
https://orcid.org/0000-0002-8053-180X

https://orcid.org/0000-0002-9246-9476

https://orcid.org/0000-0002-4122-1390

https://orcid.org/0000-0002-9066-1069

https://orcid.org/0000-0003-2121-5281

https://orcid.org/0000-0001-8713-405X 